\title{
NOTE ON INTEGRAL CLOSURES OF SEMIGROUP RINGS
}

\author{
RYÛKI MATSUDA
}

\begin{abstract}
Let $S$ be a subsemigroup which contains 0 of a torsion-free abelian (additive) group. Then $S$ is called a grading monoid (or a $g$-monoid). The group $\left\{s-s^{\prime} \mid s, s^{\prime} \in S\right\}$ is called the quotient group of $S$, and is denored by $q(S)$. Let $R$ be a commutative ring. The total quotient ring of $R$ is denoted by $q(R)$. Throught the paper, we assume that a $g$-monoid properly contains $\{0\}$. A commutative ring is called a ring, and a non-zero-divisor of a ring is called a regular element of the ring.

We consider integral elements over the semigroup $\operatorname{ring} R[X ; S]$ of $S$ over $R$. Let $S$ be a $g$ monoid with quotient group $G$. If $n \alpha \in S$ for an element $\alpha$ of $G$ and a natural number $n$ implies
\end{abstract} $\alpha \in S$, then $S$ is called an integrally closed semigroup. We know the following fact:

Theorem 1 ([G2, Corollary 12.11]). Let $D$ be an integral domain and $S$ a g-monoid. Then $D[X ; S]$ is integrally closed if and only if $D$ is an integrally closed domain and $S$ is an integrally closed semigroup.

Let $R$ be a ring. In this paper, we show that conditions for $R[X ; S]$ to be integrally closed reduce to conditions for the polynomial ring of an indeterminate over a reduced total quotient ring to be integrally closed (Theorem 15). Clearly the quotient field of an integral domain is a von Neumann regular ring. Assume that $q(R)$ is a von Neumann regular ring. We show that $R[X ; S]$ is integrally closed if and only if $R$ is integrally closed and $S$ is integrally closed (Theorem $20)$. Let $G$ be a $g$-monoid which is a group. If $R$ is a subring of the $\operatorname{ring} T$ which is integrally closed in $T$, we show that $R[X ; G]$ is integrally closed in $T[X ; S]$ (Theorem 13). Finally, let $S$ be sub- $g$-monoid of a totally ordered abelian group. Let $R$ be a subring of the ring $T$ which is integrally closed in $T$. If $g$ and $h$ are elements of $T[X ; S]$ with $h$ monic and $g h \in R[X ; S]$, we show that $g \in R[X ; S]$ (Theorem 24).

\section{General Rings}

Let $G$ be an abelian group. Then a maximal number $n$ so that there exist a set of $n$-elements in $G$ which is independent over $\mathbf{Z}$ is called the torsion-free rank of $G$, and is denoted by t.f.r. $(G)$.

Lemma 2. Let $G$ be a $g$-monoid which is finitely generated and with t.f.r. $(G)=$ $n$. Then $R[X ; G]$ is isomorphic to the ring $R\left[X_{1}, X_{1}^{-1}, \ldots, X_{n}, X_{n}^{-1}\right]$ over $R$ (where $X_{1}, \ldots, X_{n}$ are indeterminates).

Received April 21, 1999.

2000 Mathematics Subject Classification. Primary 13B22; secondary 20M25.

Key words and phrases. Semigroup ring, integral closure, von Neumann regular ring. 
Proof. Since $G$ is torsion-free, $G$ is the direct sum $\mathbf{Z} u_{1} \oplus \cdots \oplus \mathbf{Z} u_{n}$ for some elements $u_{i}$ of $G$. Set $\sigma\left(X_{i}\right)=X^{u_{i}}$ for each $i$. Then we have an isomorphism $\sigma$ of $R\left[X_{1}, X_{1}^{-1}, \ldots, X_{n}, X_{n}^{-1}\right]$ onto $R[X ; G]$.

Lemma 3. Let $f$ be an element of $R[X ; S]$. Then $f$ is a zero-divisor of $R[X ; S]$ if and only if there exists a non-zero element a of $R$ such that $a a_{i}=0$ for every coefficient $a_{i}$ of $f$.

Proof. (1) Let $f$ be a zero-divisor in $R\left[X_{1}, X_{1}^{-1}, \ldots, X_{n}, X_{n}^{-1}\right]$, then there exists a non-zero element $a$ of $R$ such that $a f=0$. For, there exists a natural number $m$ such that $X^{m} f$ is a zero-divisor in $R\left[X_{1}, \ldots, X_{n}\right]$. By $[G 1,(28.7)$ Proposition], there exists a non-zero element $a$ of $R$ such that $a X^{m} f=0$, and hence $a f=0$.

(2) Let $f$ be a zero-divisor in $R[X ; S]$. Then $f$ is a zero-divisor in $R[X ; G]$, where $G=q(S)$. There exists a finitely generated subgroup $H$ of $G$ such that $f$ is a zero-divisor in $R[X ; H] . R[X ; H]$ is isomorphic to the ring $R\left[X_{1}, X_{1}^{-1}, \ldots, X_{n}, X_{n}^{-1}\right]$ for some $n$ by Lemma 2. By (1), there exists a non-zero element $a$ of $R$ such that $a f=0$.

Let $S$ be a $g$-monoid with quotient group $G$, and $T$ a sub- $g$-monoid of $S$ with quotient group $H \subset G$. Let $R$ be a ring with total quotient ring $K$. Then all of $R, R[X ; T]$, $K[X ; T]$ and $q(R[X ; T])$ are canonically regarded as subrings of $q(R[X ; S])$ by Lemma 3 .

Lemma 4([G1, (10.2) Proposition] $]$. Let $R$ be a subring of the ring $T$, and $A$ be the integral closure of $R$ in $T$. If $N$ is a multiplicative system in $R$, then the quotient ring $A_{N}$ is the integral closure of $R_{N}$ in $T_{N}$.

Lemma 5. If $R[X ; S]$ is integrally closed, then $R$ is integrally closed.

Proof. Let $x$ be an element of $K=q(R)$ which is integral over $R$. Since $x$ is an element of $q(R[X ; S])$ which is integral over $R[X ; S], x$ belongs to $R[X ; S]$, and hence $x \in R$. Therefore $R$ is integrally closed.

Lemma 6. If $R[X ; S]$ is integrally closed, then $S$ is integrally closed.

Proof. Let $\alpha$ be an element of $q(S)$ which is integral over $S$. Since $n \alpha \in S$ for some natural number $n$, we have an integral equation of the element $X^{\alpha}$ of $q(R[X ; S])$ over $R[X ; S]$. It follows that $X^{\alpha} \in R[X ; S]$, and hence $\alpha \in S$. Therefore $S$ is integrally closed.

Lemma 7. If $R[X ; S]$ is integrally closed, then $R$ is a reduced ring.

Proof. Suppose that $R$ has a non-zero nilpotent $a$. Take non-zero $\alpha$ of $S$. Then $1+X^{\alpha}$ is a regular element of $R[X ; S]$ by Lemma 3. Then $a /\left(1+X^{\alpha}\right)$ is a nilpotent of $q(R[X ; S])$, and $a /\left(1+X^{\alpha}\right) \notin R[X ; S]$; a contradiction. Hence $R$ is reduced.

Lemma 8. Let $G$ be a g-monoid which is a group. Let $\left\{H_{\lambda} \mid \lambda\right\}$ be the set of finitely generated non-zero subgroups of $G$. Let $A_{\lambda}$ be the integral closure of $R\left[X ; H_{\lambda}\right]$. Then $\cup A_{\lambda}$ is the integral closure of $R[X ; G]$. 
Proof. Let $F$ be an element of $q(R[X ; G])$ which is integral over $R[X ; G]$. There exists a finitely generated subgroup $H$ of $G$ such that $F$ is an element of $q(R[X ; H])$ and $F$ is integral over $R[X ; H]$. Then we have $H=H_{\lambda}$ for some $\lambda$, and $F \in A_{\lambda}$. Hence the integral closure of $R[X ; G]$ is $\cap A_{\lambda}$.

Lemma 9([BCL, Lemma 1$])$. Let $G$ be a g-monoid which is a group, and $H$ a nonzero subgroup of $G$. Then $R[X ; G]$ is a free $R[X ; H]$-module. Let $\left\{\alpha_{\lambda} \mid \lambda\right\}$ be a system of complete representatives of $G$ modulo $H$. Then $\left\{X^{\alpha_{\lambda}} \mid \lambda\right\}$ is a free basis of $R[X ; G]$ over $R[X ; H]$.

Proposition 10. Let $G$ be a g-monoid which is a group. Then $R[X ; G]$ is integrally closed if and only if, for every finitely generated non-zero subgroup $H$ of $G, R[X ; H]$ is integrally closed.

Proof. The sufficiency follows from Lemma 8.

The necessity: Let $F$ be an element of $q(R[X ; H])$ which is integral over $R[X ; H]$. Since $R[X ; G]$ is integrally closed, we have $F \in R[X ; G]$. Lemma 9 implies that $F \in$ $R[X ; H]$. Hence $R[X ; H]$ is integrally closed.

Lemma 11. Let $X_{1}, X_{2}, \ldots$ be indeterminates. Let $R_{1}$ be the integral closure of $R\left[X_{1}, X_{1}^{-1}\right]$, let $R_{2}$ be the integral closure of $R_{1}\left[X_{2}, X_{2}^{-1}\right], R_{3}$ be the integral closure of $R_{2}\left[X_{3}, X_{3}^{-1}\right], \ldots$ Then $R_{n}$ is the integral closure of $R\left[X_{1}, X_{1}^{-1}, \ldots, X_{n}, X_{n}^{-1}\right]$.

Proof. We rely on the induction on $n$. Assume that $R_{n-1}$ is the integral closure of $R\left[X_{1}, X_{1}^{-1}, \ldots, X_{n-1}^{-1}, X_{n-1}^{-1}\right]$. Clearly $R_{n-1}\left[X_{n}, X_{n}^{-1}\right]$ is integral over $R\left[X_{1}, X_{1}^{-1}, \ldots\right.$, $\left.X_{n}, X_{n}^{-1}\right]$. Hence $R_{n}$ is integral over $R\left[X_{1}, X_{1}^{-1}, \ldots, X_{n}, X_{n}^{-1}\right]$. Let $F$ be an element of $q\left(R\left[X_{1}, X_{1}^{-1}, \ldots, X_{n}, X_{n}^{-1}\right)\right.$ which is integral over $R\left[X_{1}, X_{1}^{-1}, \ldots, X_{n}, X_{n}^{-1}\right]$. Then $F$ is integral over $R_{n-1}\left[X_{n}, X_{n}^{-1}\right]$. Hence $F \in R_{n}$. Therefore $R_{n}$ is the integral closure of $R\left[X_{1}, X_{1}^{-1}, \ldots, X_{n}, X_{n}^{-1}\right]$.

Lemmas 8 and 11 show that to determine the integral closure of $R[X ; G]$ reduces to determine the integral closures of $R^{\prime}\left[X, X^{-1}\right]$ for some ring $R^{\prime}$.

Lemma 12. $R\left[X_{1}, X_{1}^{-1}, \ldots, X_{n}, X_{n}^{-1}\right]$ is integrally closed if and only if $R\left[X_{1}, \ldots, X_{n}\right]$ is integrally closed.

Proof. The sufficiency follows from Lemma 4.

The necessity: Let $F$ be an element of $q\left(R\left[X_{1}, \ldots, X_{n}\right]\right)$ which is integral over $R\left[X_{1}, \ldots, X_{n}\right]$. We have $F \in R\left[X_{1}, X_{1}^{-1}, \ldots, X_{n}, X_{n}^{-1}\right]$ by assumption. If $F \notin R\left[X_{1}, \ldots\right.$, $\left.X_{n}\right]$, we may assume that $F=f_{d} X_{n}^{d}+f_{d+1} X_{n}^{d+1}+\cdots$, where each $f_{i} \in R\left[X_{1}, X_{1}^{-1}, \ldots\right.$, $\left.X_{n-1}, X_{n-1}^{-1}\right], f_{d} \neq 0$ and $d<0 . R$ is reduced by Lemma 7 . Hence there exists a prime ideal $P$ of $R$ such that $f_{d} \not \equiv 0 \bmod P R\left[X_{1}, X_{1}^{-1}, \ldots, X_{n-1}, X_{n-1}^{-1}\right]$. Set $q(R / P)=k$. Then there arises an element of $k\left[X_{1}, X_{1}^{-1}, \ldots, X_{n}, X_{n}^{-1}\right]-k\left[X_{1}, \ldots, X_{n}\right]$ which is integral over $k\left[X_{1}, \ldots, X_{n}\right]$; a contradiction. Therefore $R\left[X_{1}, \ldots, X_{n}\right]$ is integrally closed. 
Theorem 13. Let $G$ be a g-monoid which is a group. Let $T$ be an extension ring of the ring $R$ and let $A$ be the integral closure of $R$ in $T$. Then $A[X ; G]$ is the integral closure of $R[X ; G]$ in $T[X ; G]$.

Proof. (1) Let $X_{1}, \ldots, X_{n}$ be a finite number of indeterminates. Then $A\left[X_{1}, \ldots, X_{n}\right]$ is the integral closure of $R\left[X_{1}, \ldots, X_{n}\right]$ in $T\left[X_{1}, \ldots, X_{n}\right]$ (cf. [G1, (10.7) Theorem]).

(2) In (1), $A\left[X_{1}, X_{1}^{-1}, \ldots, X_{n}, X_{n}^{-1}\right]$ is the integral closure of $R\left[X_{1}, X_{1}^{-1}, \ldots, X_{n}, X_{n}^{-1}\right]$ in $T\left[X_{1}, X_{1}^{-1}, \ldots, X_{n}, X_{n}^{-1}\right]$. For, let $N$ be the multiplicative system in $R\left[X_{1}, \ldots, X_{n}\right]$ generated by $X_{1}, \ldots, X_{n}$. Since $A\left[X_{1}, \ldots, X_{n}\right]$ is the integral closure of $R\left[X_{1}, \ldots, X_{n}\right]$ in $T\left[X_{1}, \ldots, X_{n}\right]$ by (1), we see that the quotient ring $A\left[X_{1}, \ldots, X_{n}\right]_{N}$ is the integral closure of $R\left[X_{1}, \ldots, X_{n}\right]_{N}$ in $T\left[X_{1}, \ldots, X_{n}\right]_{N}$ by Lemma 4 . Hence $A\left[X_{1}, X_{1}^{-1}, \ldots, X_{n}, X_{n}^{-1}\right]$ is the integral closure of $R\left[X_{1}, X_{1}^{-1}, \ldots, X_{n}, X_{n}^{-1}\right]$ in $T\left[X_{1}, X_{1}^{-1}, \ldots, X_{n}, X_{n}^{-1}\right]$.

(3) Assume that $G$ is finitely generated. Then $A[X ; G]$ is the integral closure of $R[X ; G]$ in $T[X ; G]$. For, $R[X ; G]$ (resp. $A[X ; G]$ and $T[X ; G]$ ) is isomorphic to $R\left[X_{1}\right.$, $\left.X_{1}^{-1}, \ldots, X_{n}, X_{n}^{-1}\right]$ (resp. $A\left[X_{1}, X_{1}^{-1}, \ldots, X_{n}, X_{n}^{-1}\right]$ and $T\left[X_{1}, X_{1}^{-1}, \ldots, X_{n}, X_{n}^{-1}\right]$ ) for some $n$ by Lemma 2. (2) implies that $A[X ; G]$ is the integral closure of $R[X ; G]$ in $T[X ; G]$.

(4) Assume that $G$ is a $g$-monoid which is a group. Let $F=\Sigma a_{i} X^{\alpha_{i}}$ be an element of $T[X ; G]$ which is integral over $R[X ; G]$. We have $F_{m}+F_{m-1} f_{m-1}+\cdots+f_{0}=0$ for the elements $f_{i}$ of $R[X ; G]$. There exists a finitely generated subgroup $H$ of $G$ such that $F$ is integral over $R[X ; H]$ and belongs to $T[X ; H]$. (3) implies that $F \in A[X ; H]$. Hence $A[X ; G]$ is the integral closure of $R[X ; G]$ in $T[X ; G]$.

Proposition 14. $R[X ; S]$ is integrally closed if and only if $S$ is integrally closed, $R$ is integrally closed and $K[X ; G]$ is integrally closed, where $K=q(R)$.

Proof. The necessity: $S$ is integrally closed by Lemma $6 . R$ is integrally closed by Lemma $5 . K[X ; G]$ is integrally closed by Lemma 4 .

The sufficiency: Suppose that $R[X ; S]$ is not integrally closed. There exists $F \in$ $q(R[X ; S])-R[X ; S]$ which is integral over $R[X ; S]$. We have $F \in K[X ; G]$ by assumption. Then we have $F \in R[X ; G]$ by Theorem 13. Put $F=\Sigma a_{i} X^{\alpha_{i}}$. There exists $k$ such that $a_{k} X^{\alpha_{k}} \notin R[X ; S] . R$ is reduced by assumption and by Lemma 7 . Hence there exists a prime ideal $P$ of $R$ which does not contain $a_{k}$. Set $D=R / P$ and $\bar{a}_{i}=a_{i}+P \in D$ for each $i$. Then $\bar{F}=\Sigma \bar{a}_{i} X^{\alpha_{i}}$ is an element of $D[X ; G]-D[X ; S]$ which is integral over $D[X ; S]$. Then $\bar{F}$ is an element of $k[X ; G]-k[X ; S]$ which is integral over $k[X ; S]$, where $k=q(D)$. This contradicts to Theorem 1 .

Let $K$ be total quotient ring. We will denote the total quotient ring $q\left(K\left[X_{1}, \ldots, X_{n}\right]\right)$ of $K\left[X_{1}, \ldots, X_{n}\right]$ by $K\left(X_{1}, \ldots, X_{n}\right)$.

Theorem 15. $R[X ; S]$ is integrally closed if and only if $S$ is integrally closed, $R$ is integrally closed, $K\left[X_{1}\right]$ is integrally closed and $K\left(X_{1}, \ldots, X_{n-1}\right)\left[X_{n}\right]$ is integrally closed for every $n$ with $n \leq t$.f.r. $(q(S))$, where $K=q(R)$. 
Proof. The necessity: $S$ is integrally closed, $R$ is integrally closed and $K[X ; G]$ is integrally closed by Proposition 14. There exists a finitely generated subgroup $H$ of $G$ such that t.f.r. $(H)=n . K[X ; H]$ is integrally closed by Proposition 10 . Hence $\left(K\left[X_{1}, X_{1}^{-1}, \ldots, X_{n-1}, X_{n-1}^{-1}\right]\right)\left[X_{n}, X_{n}^{-1}\right]$ is integrally closed. Then $K\left(X_{1}, \ldots, X_{n-1}\right)\left[X_{n}\right]$ is integrally closed by Lemma 12 .

The sufficiency: We will show that $K\left[X_{1}, X_{1}^{-1}, \ldots, X_{n}, X_{n}^{-1}\right]$ is integrally closed for every $n$ with $n \leq t$.f. $r(G)$. Suppose that $K\left[X_{1}, X_{1}^{-1}, \ldots, X_{k}, X_{k}^{-1}\right]$ is integrally closed for $k<t$.f.r. $(G)$. Then $K\left[X_{1}^{-1}, \ldots, X_{k+1}, X_{k+1}^{-1}\right]$ is integrally closed by Proposition 14 . Therefore $K[X ; G]$ is integrally closed by Proposition 10 . Then $R[X ; S]$ is integrally closed by Proposition 14.

\section{Von Neumann Regular Rings}

Let $R$ be a ring. If, for each element $a$ of $R$, there exists an element $b$ of $R$ such that $a=a^{2} b$, then $R$ is called a von Neumann regular ring. We confer [G1, §11] for von Neumann regular rings. Every field is clearly a von Neumann regular ring.

Lemma 16. If $R$ is a von Neumann regular ring, then $R[X]$ and $R\left[X, X^{-1}\right]$ are integrally closed.

Proof. Let $F$ be an element of $q(R[X])$ which is integral over $R[X]$. We have $F=f / g$, where $f$ is an element of $R[X]$ and $g$ is a regular element of $R[X] . R[X]$ is a Bezout ring, that is, every finitely generated ideal is principal by [GP, Corollary 3.1]. Hence there exist elements $h, f^{\prime}, f_{1}, g_{1}$ of $R[X]$ such that $f=h f^{\prime}, g=h g^{\prime}, h=f f_{1}+g g_{1}$, where $h$ and $g^{\prime}$ are regular. Then we have $1 / g^{\prime}=F f_{1}+g_{1}$. Hence $1 / g^{\prime}$ is integral over $R[X]$. The integral equation of $1 / g^{\prime}$ over $R[X]$ shows that $g^{\prime}$ is a unit of $R[X]$. [G1, Corollary 11.4] implies that $g^{\prime}$ is a unit of $R$. Then we have $F=f / g=f^{\prime} / g^{\prime} \in R[X]$. Therefore $R[X]$ is integrally closed.

$R\left[X, X^{-1}\right]$ is integrally closed by Lemma 12 .

Lemma 17. If $R$ is von Neumann regular ring, then $q(R[X ; G])$ is a von Neumann regular ring.

Proof. (1) Let $f=\Sigma_{0}^{n} a_{i} X^{i}$ be an element of $R[X]$. Then there exists $F \in q(R[X])$ such that $f=f^{2} F$. For the proof, we rely on the induction on $n$. Thus suppose that the assertion holds for fewer degrees. Assume that $a_{n} \neq 0$. There exists an idempotent $e$ of $R$ such that $R a_{n}=R e$. Put $e^{\prime}=1-e$. Since $\operatorname{deg}\left(f e^{\prime}\right)<n$, there exist elements $f_{1}$ and $g_{1}$ of $R[X]$ with $g_{1} e^{\prime}$ regular in $R e^{\prime}[X]$ such that $f e^{\prime}=f^{2} f_{1} e^{\prime} /\left(g_{1} e^{\prime}\right)$. Note that $f e$ is regular in $R e[X]$. We see that $g_{1} e^{\prime}+f e$ is a regular element of $R[X]$. Further we have $f=f^{2}\left(f_{1} e^{\prime}+e\right) /\left(g_{1} e^{\prime}+f e\right)$.

(2) $q\left(R\left[X_{1}, \ldots, X_{n}\right]\right)$ is von Neumann regular. For the proof, we rely on the induction on $n$. (1) implies that $q\left(R\left[X_{1}\right]\right)$ is von Neumann regular. Suppose that $q\left(R\left[X_{1}, \ldots, X_{n-1}\right]\right)$ is von Neumann regular. It follows that $q\left(q\left(R\left[X_{1}, \ldots, X_{n-1}\right]\right)\left[X_{n}\right]\right)$ is von Neumann regular. That is, $q\left(R\left[X_{1}, \ldots, X_{n}\right]\right)$ is von Neumann regular. 
(3) Let $f$ be an element of $q(R[X ; G])$. There exists a finitely generated subgroup $H$ of $G$ such that $f \in q(R[X ; H])$. Since $R[X ; H]$ is isomorphic to the $\operatorname{ring} R\left[X_{1}, X_{1}^{-1}, \ldots\right.$, $\left.X_{n}, X_{n}^{-1}\right]$ for some $n, q(R[X ; H])$ is von Neumann regular by (2). Hence there exists $F \in q(R[X ; H])$ such that $f=f^{2} F$. Therefore $q(R[X ; G])$ is von Neumann regular.

[G1, $\S 11$, Exercise 13] states that if $R$ is its own total quotient ring and if $R$ is reduced, then $R$ is 0 -dimensional. If this is the case, then $q(R[X ; G])$ is a von Neumann regular ring for every reduced ring $R$. Now we have the following,

Example 18 (Gilmer and Matsuda). Let $k$ be a field and let $X_{1}, X_{2}, \ldots$ be indeterminates.

(1) Set $R=k\left[\left[X_{1}, X_{2}, \ldots\right]\right]_{1} /\left(X_{i} X_{j} \mid i \neq j\right)$, where $k\left[\left[X_{1}, X_{2}, \ldots\right]\right]_{1}$ is the union of the ascending net of rings $k\left[\left[X_{1}, \ldots, X_{n}\right]\right]$ of all $n$. Then $R$ is its own total quotient ring and reduced. But $R$ is not 0 -dimensional.

(2) Let $R=k\left[X_{1}, X_{2}, \ldots\right] /\left(X_{i} X_{j} \mid i \neq j\right)$ and $M=\left(X_{1}, X_{2}, \ldots\right) R$. Then $R_{M}$ is its own total quotient ring and reduced. But $R_{M}$ is not 0 -dimensional.

Lemma 19. If $R$ is a von Neumann regular ring, then $R[X ; G]$ is integrally closed.

Proof. (1) $R\left[X, X^{-1}\right]$ is integrally closed by Lemma 16 .

(2) $R\left[X_{1}, X_{1}^{-1}, \ldots, X_{n}, X_{n}^{-1}\right]$ is integrally closed. For the proof, we rely on the induction on $n$. Suppose that $R\left[X_{1}, X_{1}^{-1}, \ldots, X_{n-1}, X_{n-1}^{-1}\right]$ is integrally closed. $q\left(R\left[X_{1}, \ldots, X_{n-1}\right]\right)$ is von Neumann regular by Lemma 17 . (1) implies that $q\left(R\left[X_{1}, \ldots\right.\right.$, $\left.\left.X_{n-1}\right]\right)\left[X_{n}, X_{n}^{-1}\right]$ is integrally closed. Then Proposition 14 implies that $R\left[X_{1}, X_{1}^{-1}, \ldots\right.$, $\left.X_{n-1}, X_{n-1}^{-1}\right]$ is integrally closed.

(3) Let $F$ by an element of $q(R[X ; G])$ which is integral over $R[X ; G]$. There exists a finitely generated subgroup $H$ of $G$ such that $F \in q(R[X ; H])$ and $F$ is integral over $R[X ; H]$. Since $R[X ; H]$ is isomorphic to the $\operatorname{ring} R\left[X_{1}, X_{1}^{-1}, \ldots, X_{n}, X_{n}^{-1}\right]$ for some $n$, (2) implies that $F \in R[X ; H]$. Therefore $R[X ; G]$ is integrally closed.

Theorem 20. Assume that $q(R)$ is a von Neumann regular ring. Then $R[X ; S]$ is integrally closed if and only if $S$ is integrally closed and $R$ is integrally closed.

Proof. The necessity is clear.

The sufficiency: Set $G=q(S)$ and $K=q(R)$. Then $K[X ; G]$ is integrally closed by Lemma 19. Proposition 14 implies that $R[X ; S]$ is integrally closed.

If $R$ is a domain, then $q(R)$ is clearly a von Neumann regular ring.

\section{A Theorem}

Let $G$ be a totally ordered abelian group. Let $f$ be an element of $R[X ; G]$. Put $f=a_{1} X^{\alpha_{1}}+\cdots+a_{n} X^{\alpha_{n}}$, where the $a_{i}$ are non-zero elements of $R$ and $\alpha_{1}<\cdots<\alpha_{n}$. If $a_{n}=1$, then $f$ is called monic in $R[X ; G]$. 
Lemma 21. Let $G=\mathbf{Z} \oplus \cdots \oplus \mathbf{Z}$ (the direct sum of $n$-copies of the additive group Z) with the lexicographic order, and let $X_{1}=X^{(1,0, \ldots, 0)}<\cdots<X_{n}=X^{(0, \ldots, 0,1)}$. Let $T$ be a ring and $R$ a subring of $T$ which is integrally closed in $T$. Let $g$ and $h$ are elements of $T[X ; G]$ with $h$ monic and $g h \in R[X ; G]$. Then $g \in R[X ; G]$.

Proof. We may assume that $g=g\left(X_{1}, \ldots, X_{n}\right)$ and $h=h\left(X_{1}, \ldots, X_{n}\right)$ belong to $T\left[X_{1}, \ldots, X_{n}\right]$. If $n=1$, the assertion holds by [G1, (10.4) Theorem]. We rely on the induction on $n$. Suppose that the asertion holds for $n-1$. There exists a natural number $m$ such that the coefficients of $g\left(X_{1}, \ldots, X_{n-1}, X_{n}\right)\left(\operatorname{resp} . h\left(X_{1}, \ldots, X_{n-1}, X_{n}\right)\right)$ and $g\left(X_{1}, \ldots, X_{n-1}, X_{n-1}^{m}\right)$ (resp. $\left.h\left(X_{1}, \ldots, X_{n-1}, X_{n-1}^{m}\right)\right)$ are the same and $h\left(X_{1}, \ldots\right.$, $\left.X_{n-1}, X_{n-1}^{m}\right)$ is monic. Since $g\left(X_{1}, \ldots, X_{n-1}, X_{n-1}^{m}\right) h\left(X_{1}, \ldots, X_{n-1} X_{n-1}^{m}\right) \in R\left[X_{1}, \ldots\right.$, $\left.X_{n-1}\right]$, we have $g\left(X_{1}, \ldots, X_{n-1}, X_{n-1}^{m}\right) \in R\left[X_{1}, \ldots, X_{n-1}\right]$ by hypothesis. Hence $g \in$ $R[X ; G]$.

Lemma 22. Let $G$ be a finitely gnerated subgroup of the totally ordered abelian group R. Let $T$ be a ring and $R$ a subring which is integrally closed in $T$. If, for elements $g$ and $h$ of $T[X ; G]$ with $h$ monic, gh belongs to $R[X ; G]$, then $g$ belongs to $R[X ; G]$.

Proof. There exist real numbers $\pi_{1}, \ldots, \pi_{n}$ so that $G=Z \pi_{i}+\cdots+Z \pi_{n}$ and $\left\{\pi_{1}, \ldots, \pi_{n}\right\}$ is independent over $\mathbf{Z}$. We may assume that $0<\pi_{1}<\cdots<\pi_{n}$. Set $X_{i}=X^{\pi_{i}}$ for each $i$. We may assume that $g=g\left(X_{1}, \ldots, X_{n}\right)$ and $h=h\left(X_{1}, \ldots, X_{n}\right)$ are elements of $T\left[X_{1}, \ldots, X_{n}\right]$. Arrange the powers of $g$ and $h$ as follows: $\Sigma k_{1 i} \pi_{i}<\cdots<$ $\Sigma k_{m i} \pi_{i}$. It follows that $\Sigma k_{1 i} \pi_{i} / \pi_{1}<\cdots<\Sigma k_{m i} \pi_{i} / \pi_{1}$.

Since all of $\pi_{2} / \pi_{1}, \ldots, \pi_{n} / \pi_{1}$ are irrational, there exist positive rational numbers $a_{1}=1, a_{2}, \ldots, a_{n}$ such that $\Sigma k_{1 i} a_{i}<\cdots<\Sigma k_{m i} a_{i}$. Hence there exist positive integers $p_{1}, \ldots, p_{n}$ such that $\Sigma k_{1 i} p_{i}<\cdots<\Sigma k_{m i} p_{i}$. Note that, if $\left(k_{1}, \ldots, k_{n}\right) \neq\left(l_{1}, \ldots, l_{n}\right)$, then $\Sigma k_{i} \pi_{i} \neq \Sigma l_{i} \pi_{i}$. Then the coefficients of $g\left(X_{1}, \ldots, X_{n}\right)\left(\right.$ resp. $\left.h\left(X_{1}, \ldots, X_{n}\right)\right)$ and $g\left(Y^{p 1}, \ldots, Y^{p n}\right)$ (resp. $\left.h\left(Y^{p 1}, \ldots, Y^{p n}\right)\right)$ are the same and $h\left(Y^{p 1}, \ldots, Y^{p n}\right)$ is monic (where $Y$ is an anothr indeterminate). Since $g\left(Y^{p 1}, \ldots, Y^{p n}\right) h\left(Y^{p 1}, \ldots, Y^{p n}\right) \in R[Y]$, we have $g\left(Y^{p 1}, \ldots, Y^{p n}\right) \in R[Y]$. Hence $g \in R[X ; G]$.

Theorem 23. Let $S$ be a sub-g-monoid of a totally ordered abelian group. Let $R$ be a subring of the ring $T$, and let $A$ be the integral closure of $R$ in $T$. If, for elements $g$ and $h$ of $T[X, S]$ with $h$ monic, gh belongs to $R[X, S]$, then $g \in A[X ; S]$.

Proof. We may assume that $G=S$ is a finitely generated group and $R$ is integrally closed in $T$. We may assume that $G=H_{1} \oplus \cdots \oplus H_{k}$ with the lexicographic order, where the $H_{i}$ are non-zero subgroups of the totally ordered abelian group $\mathbf{R}$. Since $G$ is finitely generated, we may use a similar argument to $[\mathrm{ZS}, \mathrm{VI},(\mathrm{A})]$. We rely on the induction on t.f.r. $(G)$. Assume that t.f.r. $(G)=r$, and suppose that the assertion holds for fewer torsion-free ranks. The powers of $g$ and $h$ are of the form $\left(h_{1}, \ldots, h_{k}\right)$ for the $h_{i} \in H_{i}$. Suppose that each $k$-component $h_{k}$ is zero for every power of $g$ and $h$. Then we have $g \in R[X ; G]$ by induction. Suppose that $h_{k}$ is non-zero for some power of $g$ or $h$. Let $H_{i}=Z \pi_{i 1}+\cdots+Z \pi_{i n(i)}$, where the set $\left\{\pi_{i 1}, \ldots, \pi_{i n(i)}\right\}$ is independent over $\mathbf{Z}$ for each 
$i$. We may assume that $0<\pi_{i 1}<\cdots<\pi_{i n(i)}$ for each $i$. Put $X_{i j}=X^{\left(0, \ldots, \pi_{i j}, 0, \ldots\right)}$ for each $i$ and $j$. We may assume that $g$ and $h$ belong to $T\left[X_{11}, \ldots, X_{k n(k)}\right]$.

The case of $n(i)=1$ for each $i$ : Then $G$ is order-isomorphic to $Z \oplus \cdots \oplus Z$ (the didrect sum of $k$-copies of $\mathbf{Z}$ ) with the lexicographic order. By Lemma 21, we have $g \in R[X ; G]$.

The case of $n(i)>1$ for some $i$ : For each $l$, arrange the $l$-components of $g$ and $h$ as follows: $\Sigma k_{l 1 i} \pi_{l i}<\Sigma k_{l 2 i} \pi_{l i}<\cdots<\Sigma k_{l m(l) i} \pi_{l i}$. Then, as in the proof of Lemma 22, there exist positive integers $p_{i j}$ such that $\Sigma k_{l 1 i} p_{l i}<\Sigma k_{l 2 i} p_{l i}<\cdots<\Sigma k_{l m(l) i} p_{l i}$. Let $Y_{1}, \ldots, Y_{k}$ be another indeterminates. Then the coefficients of $g=g\left(X_{11}, \ldots, X_{k n(k)}\right)$ (resp. $\quad h=h\left(X_{11}, \ldots, X_{k n(k)}\right)$ and $g\left(Y_{1}^{p_{11}}, \ldots, Y_{1}^{p_{1 n}(1)}, \ldots, Y_{k}^{p_{k 1}}, \ldots, Y_{k}^{p_{k n}(k)}\right)$ (resp. $\left.\left.h\left(Y_{1}^{p_{11}}, \ldots, Y_{1}^{p_{1 n}(1)}, \ldots, Y_{k}^{p_{k 1}}, \ldots, Y_{k}^{p_{k n}(k)}\right)\right)\right)$ are the same and $h\left(Y_{1}^{p_{11}}, \ldots, Y_{1}^{p_{1 n}(1)}, \ldots\right.$, $\left.Y_{k}^{p_{k 1}}, \ldots, Y_{k}^{p_{k n}(k)}\right)$ is monic. Since $g\left(Y_{1}^{p_{11}}, \ldots, Y_{1}^{p_{1 n}(1)}, \ldots, Y_{k}^{p_{k 1}}, \ldots, Y_{k}^{p_{k n}(k)}\right) h\left(Y_{1}^{p_{11}}, \ldots\right.$, $\left.Y_{1}^{p_{1 n}(1)}, \ldots, Y_{k}^{p_{k 1}}, \ldots, Y_{k}^{p_{k n}(k)}\right) \in R\left[Y_{1}, \ldots, Y_{k}\right]$, we have $g\left(Y_{1}^{p_{11}}, \ldots, Y_{1}^{p_{1 n}(1)}, \ldots, Y_{k}^{p_{k 1}}\right.$, $\left.\ldots, Y_{k}^{p_{k n}(k)}\right) h\left(Y_{1}^{p_{11}}, \ldots, Y_{1}^{p_{1 n}(1)}, \ldots, Y_{k}^{p_{k 1}}, \ldots, Y_{k}^{p_{k n(k)}}\right) \in R\left[Y_{1}, \ldots, Y_{k}\right]$. Hence $g\left(Y_{1}^{p_{11}}\right.$, $\left.\ldots, Y_{1}^{p_{1 n}(1)}, \ldots, Y_{k}^{p_{k 1}}, \ldots, Y_{k}^{p_{k n}(k)}\right) \in R\left[Y_{1}, \ldots, Y_{k}\right]$. Therefore $g \in R[X ; G]$.

\section{References}

[1] [BCL] J. Brewer, D. Costa and E. Lady, Prime ideals and localization in commutative group rings, J. Algebra, 34(1975), 300-308.

[2] [G1] R. Gilmer, Multiplicative Ideal Theory, Marcel Dekker, 1972.

[3] [G2] R. Gilmer, Commutative Semigroup Rings, The Univ. Chicago Press, 1984.

[4] [GP] R. Gilmer and T. Parker, Semigroup rings as Prüfer rings, Duke Math. J., 41(1974), 219-230.

[5] [ZS] O. Zariski and P. Samuel, Commutative Algebra II, Van Nostrand, 1960.

Department of Mathematics, Ibaraki University, Mito, Ibaraki 310, Japan. 